\title{
A multi-centre study on quality of life and absenteeism in patients with CRS referred for endoscopic surgery*
}

\author{
Pernilla Sahlstrand-Johnson ${ }^{1}$, Bodil Ohlsson ${ }^{2}$, Christian von Buchwald ${ }^{3}$, \\ Magnus Jannert ${ }^{1}$, Marianne Ahlner-Elmqvist ${ }^{4}$ \\ 1 Department of Oto-Rhino-Laryngology, Head and Neck Surgery, Faculty of Medicine, Lund University, \\ Skåne University Hospital, Malmö, Sweden \\ 2 Department of Clinical Sciences, Division of Gastroenterology, Faculty of Medicine, Lund University, Skåne \\ University Hospital, Malmö, Sweden \\ 3 Department of Otolaryngology, Head and Neck Surgery, Rigshospitalet, Copenhagen University Hospital, \\ Copenhagen, Denmark \\ 4 Department of Health Sciences, Faculty of Medicine, Lund University, Lund, Sweden
}

SUMMARY

\section{INTRODUCTION}

The impact of rhinosinusitis on health-related quality of life (HRQOL) has proved to be significant, and greater than that of other chronic conditions, such as chronic obstructive pulmonary disease and angina pectoris ${ }^{(1)}$. Chronic rhinosinusitis (CRS) is a common condition with a reported prevalence of $9.3 \%$ in Europe ${ }^{(2)}$. In the USA, CRS results in more than 200,000 surgical procedures annually ${ }^{(3)}$. The economic impact of rhinosinusitis is significant, since the disease results in both direct health care costs, such as medication, hospitalisation, physician visits and surgery, and indirect costs such as disabil- ity and absenteeism from work, leading to loss of productivity. For example, it has been reported that approximately 73 million days of restricted activity were related to sinusitis in the USA in $1992^{(4)}$.

A lack of well-defined criteria defining rhinosinusitis has previously prevented reliable descriptive and outcome studies in patients with sinus disease. The first European Position Paper on Rhinosinusitis and Nasal Polyps (EP ${ }^{3} \mathrm{OS}$ ) was published in $2005^{(5)}$, and presented evidence-based knowledge on, and definitions of, rhinosinusitis. A revision followed in 2007 with 
criteria for diagnosing acute and chronic rhinosinusitis ${ }^{(6)}$. Interest in outcome studies on rhinosinusitis, not least assessments of HRQOL, has grown during the past decade, resulting in a number of publications on this subject. The most extensive outcome study so far is the National Comparative Audit of Surgery for Chronic Rhinosinusitis and Nasal Polyposis, in the UK, including 3128 patients ${ }^{(7)}$. Preoperative HRQOL scoring can predict how the patient will perceive the result of sinus surgery ${ }^{(8)}$, and validated HRQOL instruments should be used when assessing the outcome of functional endoscopic sinus surgery (FESS) ${ }^{(9)}$. Tomassen et al. have shown that the $\mathrm{EP}^{3} \mathrm{OS}$ criteria are suitable for the assessment of geographic variation in the prevalence of CRS ${ }^{(2)}$. To the best of the authors' knowledge, no European studies on the effect of CRS on absenteeism, based on the definition of the condition in the $\mathrm{EP}^{3} \mathrm{OS}$ document, have been published.

The aims of the present study were to obtain data on HRQOL, psychiatric morbidity and absenteeism in patients referred for FESS with the diagnoses RARS, CRS+NP and CRS-NP, in a large, prospective multi-centre study. We also wanted to evaluate whether there was a difference in HRQOL scoring among these subgroups of patients with chronic sinus disease.

\section{MATERIAL AND METHODS}

Study population

Patients aged $\geq 18$ years with a diagnosis of RARS, CRS+NP or CRS-NP referred for FESS at 10 hospitals (five university and five regional hospitals) in Sweden during 2008 - 2009 were prospectively enrolled. Data collection did not start until in 2009 in one of the hospitals. The diagnostic criterion for acute rhinosinusitis was defined according to $\mathrm{EP}^{3} \mathrm{OS}$ as the presence of two or more symptoms, one of which should be nasal congestion/obstruction/ blockage or nasal discharge (anterior/ posterior nasal drip), and/or facial pain/pressure, and/or reduction or loss of smell for less than 12 weeks, with an increase in symptoms after five days, or persistent symptoms after 10 days (6). Patients were enrolled with a diagnosis of RARS if they fulfilled the following two criteria: 1) a clinical history of four or more recurrent acute bouts of rhinosinusitis in the previous 12-month period, and 2) a paucity of sinonasal symptoms during intervals between acute episodes ${ }^{(10,11)}$. The diagnostic criterion for CRS with or without NP was defined as the presence of two or more symptoms, one of which should be nasal congestion/obstruction/blockage or nasal discharge (anterior/posterior nasal drip), and/or facial pain/pressure, and/or reduction or loss of smell for more than 12 weeks. Additionally, there should be endoscopic signs and/or radiological signs of CRS with or without nasal polyps on computed tomography (CT), according to $\mathrm{EP}^{3} \mathrm{OS}{ }^{(6)}$. Exclusion criteria were pregnancy, gross immunodeficiency, congenital mucociliary problems, cystic fibrosis and systemic vasculitis, and granulomatous diseases, as these conditions may induce sinus disease and affect HRQOL. The ability to understand the Swedish language was mandatory for inclusion.

\section{Study design}

The patients were examined at the hospital by sinus surgeons, who diagnosed RARS, CRS+NP or CRS-NP, and referred the patients for surgery. All patients had previously received adequate medical treatment according to $\mathrm{EP}^{3} \mathrm{OS}$ guidelines ${ }^{(6)}$ without satisfactory response, before being referred for surgery; i.e. the patients with CRS-NP had received nasal steroid and nasal lavage for at least three months, and patients with CRS+NP had received nasal steroids for at least three months and one or more courses of oral steroids.

The patients included in the study were sent questionnaires (see below) preoperatively together with information on their appointment for surgery (usually 2 - 4 weeks prior to surgery). All patients also completed a form on tobacco habits, work, current health status and allergy, medication, and the onset of sinus problems. The patients were also asked how many days they thought they had been absent from work (absenteeism) due to sinus problems during the past 12 months. The possible alternatives were: 0 days, 1 - 7 days, 8 - 14 days, 15 - 30 days or $>30$ days. A clinician-reported data form was completed by the surgeons at the time of surgery. All the questionnaires were returned to the study coordinator, and the data recorded anonymously in a computer database.

The Local Ethics Committee of Lund University approved the study protocol. At the time of preoperative assessment the patients who met the entry criteria received written and verbal information about the study, and gave their informed written consent to participate. They were also informed that participation in the survey was voluntarily, would not mean any change in care or surgery procedure, and that the results of the study would be treated anonymously.

\section{Endoscopic polyp grading}

Nasal endoscopy was performed at surgery and polyps graded in each nasal cavity according to an established classification system $^{(6)}(0=$ no visible polyps; $1=$ polyps confined to middle meatus; 2 = polyps below level of middle turbinate but not causing total obstruction; 3 = polyps causing total nasal obstruction). A topical anaesthetic and a decongestant were applied prior to performing endoscopy.

The cohort was further divided into subgroups based on the polyp grading above.

- 'NP severity' - to be able to analyse whether polyps outside the middle meatus affected HRQOL scoring; group $\mathrm{A}=$ polyp extent maximum 1 on both sides; and group $\mathrm{B}=$ polyp extent 2 - 3 on at least one side.

- 'NP side'- to determine whether the amount of polyps on one side of the nose was of importance in HRQOL scoring: $\mathrm{I}=$ polyp extent 0 on both sides or at most 1 on one side; II = polyp extent 0 on one side and 2 or 3 on the other; III = polyp extent 1 on both sides, or polyp extent 1 on one side and 2-3 on the other; IV = polyp extent 2 on both sides or polyp extent 2 on one side and 3 on the other; and $\mathrm{V}=$ polyp extent 3 on both sides. 


\section{Questionnaires}

\section{Sinonasal Outcome Test (SNOT-22)}

SNOT-22 is a HRQOL questionnaire focused on sinonasal conditions. It contains 22 nose, sinus, and general items and is a modification of SNOT-20 developed by Piccirillo ${ }^{(12)}$. In SNOT-22 the scoring has been simplified by removing the importance rating, and adding two items: nasal blockage and loss of sense of taste and smell. Morley and Sharp concluded in $2006^{(13)}$ that SNOT-22 was the most suitable disease-specific instrument for use in CRS, and it has subsequently been validated and found to be easy to use ${ }^{(14)}$. SNOT-22 is today one of the most frequently used survey instruments in sinonasal research, and is available in several languages, for example Czech ${ }^{(15)}$, Chinese ${ }^{(16)}$, German ${ }^{(17)}$ and Danish ${ }^{(18)}$, and now also in Swedish. The theoretical range of the score is $0-110$, with lower scores implying a better HRQOL. The minimally important difference when using the questionnaire is $8.9^{(14)}$, i.e. a change of less than 9 points cannot be perceived as a real improvement or impairment by the patient. The procedure of forward and backward translation from English to Swedish of SNOT-22 was performed according to accepted international criteria before using the questionnaire in this study ${ }^{(19)}$.

\section{6-item short-form questionnaire ( $S F$-36)}

SF-36 is an extensively used HRQOL instrument, which provides reproducible, reliable data on large populations, and has been shown to be useful as a global health monitor in clinical practice. It is available in Swedish ${ }^{(20)}$ and reference data are available for many different conditions. The SF-36 questionnaire is divided into eight subscales of general health and ordered according to the degree to which they measure physical vs. mental health. These subscales are physical functioning (PF), role functioning-physical (RP), bodily pain (BP), general health $(\mathrm{GH})$, vitality (VT), social functioning (SF), role functioning-emotional (RE), and mental health (MH). Two additional dimensions can be calculated, namely physical (PCS) and emotional health (MCS), based on weighting of the importance of the eight subscales. The raw data are recoded when analysed; high scores imply a better HRQOL and the maximum score is 100 .

\section{Hospital Anxiety and Depression Scale (HAD)}

The HAD scale screens for psychiatric morbidity in patients with somatic disease. This HRQOL instrument contains 14 multiple-choice items, seven for anxiety and seven for depression, and has been constructed so that somatic questions are avoided ${ }^{(21)}$. It is a well-documented questionnaire, and has been used in many different patient groups, for example, in patients with head and neck cancer ${ }^{(22)}$, but only sparsely in patients with rhinosinusitis. The cut-offs are $>7$ points for possible psychiatric illness and $>10$ points for probable psychiatric illness ${ }^{(21)}$; the higher the score, the more likely the patient is to suffer from psychiatric morbidity.

\section{Visual analogue scale (VAS)}

The degree or strength of symptoms can be estimated using a visual analogue scale, VAS, which provides a subjective assessment of the symptoms. The VAS is a psychometric response scale usually represented by a horizontal line, $10 \mathrm{~cm}$ in length, anchored by word descriptors at each end. The patient indicates the position on the line that they feel represents their perception of their current state. Lim et al. have considered the relationship between subjective assessment instruments in CRS and found that 'mild' equates to a VAS of $\leq 3$, 'moderate' to $>$ 3-7 and 'severe' to $>7^{(23)}$. The question asked was: 'How troublesome are your symptoms of rhinosinusitis?' When this study was initiated, the use of the VAS to assess combined sinonasal symptoms had not been validated, and the correlation of the results with HRQOL had not been investigated.

\section{Statistical analysis}

Statistical analysis was performed with SPSS 18 (Statistical Package for the Social Sciences). The Kruskal-Wallis test and the Mann-Whitney U-test were used to compare groups. Values are expressed as the median with range or mean \pm standard deviation (SD), as well as confidence intervals (CI). One sample t-test was used to compare the SF-36 scores in our cohort with that of the normal Swedish population. Spearman's rank correlation coefficients were calculated to study correlations. Differences with a $\mathrm{p}$ value $\leq 0.05$ were considered statistically significant. We used simple mean imputation for missing data, when at least 50 percent of the items had been completed. This means that the mean of the values of the completed item is used as the value of the missing data. This method has been proven to be best for HRQOL instruments that use unweighted sum scores ${ }^{(19)}$. For SF-36, it was required that $50 \%$ of the items in the same subscale had to be competed for simple mean imputation to be used.

\section{RESULTS}

\section{Patient characteristics}

A surgeon-completed clinical data form was returned for 237 patients. Of these, the preoperative questionnaires were completed by 220 patients. Thirteen patients were excluded because they fulfilled the exclusion criteria or did not have the correct diagnosis, leaving 207 patients. Additional seventynine possible patients could not be included due to administrative difficulties, e.g. we did not receive information about surgery in time to send out the questionnaire, or it was not possible to contact the patient. The patient characteristics and division into the rhinosinusitis subgroups are given in Table 1. The median age of all patients was $48(18-85)$ years. The median age of patients with RARS was 40 (20 - 84) years, with CRS+NP $54(20$ - 85) years and CRS-NP $45(18$ - 79) years. The reported median duration of sinus problems was 59 (1 904) months. Fifty-two patients $(25 \%)$ had at least one other form of co-morbidity, specified as heart or lung disease, hypertension, diabetes mellitus, rheumatic diseases, malignancies or depression. Ten percent of the patients with nasal polyps suffered from Samter's triad (nasal polyps, asthma and hypersensitivity to acetyl salicylic acid). 


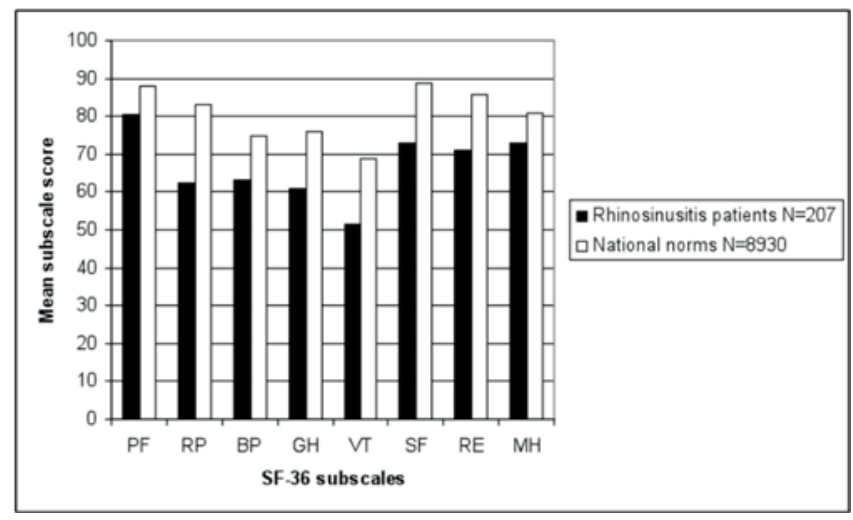

Figure 1. SF-36 health profiles for rhinosinusitis patients and national norms. High score represent high level of functioning.

Table 1. Patient-reported characteristics.

\begin{tabular}{lc}
\hline Variable & $\mathrm{n}(\%)$ \\
\hline Gender & $122(59)$ \\
Men & $85(41)$ \\
Women & 48 years \\
Age median & \\
Rhinosinusitis diagnosis & \\
$\quad$ Recurrent acute rhinosinusitis & $34(16)$ \\
$\quad$ Chronic rhinosinusitis with nasal polyps & $135(65)$ \\
$\quad$ Chronic rhinosinusitis without nasal polyps & $38(18)$ \\
Previous functional endoscopic sinus surgery & \\
$\quad$ Recurrent acute rhinosinusitis & $0(0)$ \\
Chronic rhinosinusitis with nasal polyps & $43(34)$ \\
Chronic rhinosinusitis without nasal polyps & $8(26)$ \\
Gainfully employed & $155(75)$ \\
Nicotine habits & \\
Smoker & $22(11)$ \\
Snuff user & $32(15)$ \\
Smoker and/or snuff user & $42(20)$ \\
Allergic rhinitis & $52(25)$ \\
Asthma & $54(26)$ \\
Acetyl salicylic acid intolerance & $24(12)$ \\
\hline
\end{tabular}

n: number of patients (207)

\#Surgeon-reported

\section{$\underline{S F-36}$}

The scoring on all eight subscales and in the two domains was significantly lower in our cohort $(p<0.0001)$ than the corresponding normative values of the Swedish population (Figure 1) ${ }^{(24)}$. Women scored statistically significantly lower than men on all subscales except MH and MCS. The significant difference between our rhinosinusitis population and the normative data remained after the data were gender-adjusted. There were significant differences in the BP and MCS scores between the three subgroups (Table 3).

\section{$\underline{H A D}$}

The results for the HAD scale for the whole study population are given in Table 4. The numbers of patients with scores indicating probable or possible cases of anxiety disorder were $52(25 \%)$ and depression disorder $28(14 \%)$, respectively. Fiftyseven $(28 \%)$ patients scored $>7$ points in the HAD scale. There was no statistically significant difference in the prevalence of depression or anxiety between men and women. Nor did age significantly affect the HAD scores. Patients with RARS had significantly higher anxiety scores than patients with CRS+NP $(p=0.006)$, see Table 4 .

\section{$\underline{V A S}$}

The mean VAS score for combined sinonasal symptoms for all patients was $67 \pm 24$. There was no statistically significant difference in VAS scores between men and women, nor between the three rhinosinusitis groups. According to the VAS classification, $9.8 \%$ of the patients had mild, $37.6 \%$ moderate and $52.7 \%$ severe disease. There was no statistically significant difference in any HRQOL or VAS score when comparing revision cases $(n=51)$ with patients who had not previously undergone sinus surgery.

\section{Correlations between SNOT-22, SF-36, HAD and VAS scores}

A significant positive correlation was found between the VAS scores and the total SNOT-22 score $(r=0.477, \mathrm{p}<0.0001)$, and between the VAS classification (mild, moderate and severe) and the total SNOT-22 score $(r=0.389, p<0.0001)$. The mean SNOT score for the mild disease group was $26 \pm 16$, for the moderate group $48 \pm 18$ and for the severely diseased group 59 \pm 21 . The patients in the different VAS groups (mild, moderate and severe) had significantly different total SNOT-22 ( $\mathrm{p}<$ $0.0001)$ scores, all eight SF-36 subscales $(\mathrm{p}<0.0001)$ and HAD scores $(\mathrm{p}<0.0001)$. Moreover, a negative correlation was seen between all eight domains of the SF-36 questionnaire and the total SNOT score $(r=-0,702$ to $-0.516, p<0.0001)$, indicating good consistency in the scoring systems. A positive correlation was found between the depression and anxiety classification (HAD) and the total SNOT-22 score $(r=0.460, p<0.0001 ; \mathrm{r}$ $=0.541, \mathrm{p}<0.000$, respectively) and between the HAD classification and the VAS score $(r=0.270, p<0.0001 ; r=0.239$, $\mathrm{p}=0.001$, respectively). The scores for the MH subscale of the SF-36 questionnaire were strongly correlated to both anxiety $(r=-0.756, p<0.0001)$ and depression scores obtained from consistency. 
Table 2. SNOT-22 scores of all patients.

\begin{tabular}{|c|c|c|c|c|c|}
\hline & $\begin{array}{l}\text { All patients }^{\mathrm{a}} \\
\qquad(\mathrm{n}=180)\end{array}$ & $\begin{array}{l}\text { Recurrent acute } \\
\text { rhinosinusitis ( } \\
=32 \text { ) }\end{array}$ & $\begin{array}{l}\text { Chronic rhinosi- } \\
\text { nusitis with NP } \\
\quad(\mathrm{n}=112)\end{array}$ & $\begin{array}{l}\text { Chronic rhinosi- } \\
\text { nusitis without } \\
\text { NP }(\mathrm{n}=36)\end{array}$ & $p$ value $^{b}$ \\
\hline & Mean & Mean & Mean & Mean & \\
\hline Total SNOT-22 score & 51.8 & 57.8 & 49.0 & 54.5 & 0.085 \\
\hline \multicolumn{6}{|l|}{ SNOT-22 questions } \\
\hline 1. Need to blow nose & 3.5 & 3.3 & 3.6 & 3.4 & 0.190 \\
\hline 2. Sneezing & 2.1 & 1.9 & 2.2 & 2.1 & 0.399 \\
\hline 3. Runny nose & 2.9 & 2.3 & 3.1 & 2.6 & 0.022 \\
\hline 4. Blockage/congestion of nose & 3.9 & 3.9 & 3.9 & 3.9 & 0.944 \\
\hline 5. Loss of sense of taste/smell & 3.4 & 2.3 & 4.0 & 2.7 & 0.0001 \\
\hline 6. Cough & 1.7 & 1.9 & 1.5 & 2.4 & 0.009 \\
\hline $\begin{array}{l}\text { 7. Post-nasal discharge (dripping at the } \\
\text { back of your nose) }\end{array}$ & 2.8 & 3.2 & 2.7 & 3.0 & 0.191 \\
\hline 8. Thick nasal discharge & 2.7 & 2.8 & 2.5 & 3.0 & 0.357 \\
\hline 9. Ear fullness & 2.3 & 2.8 & 2.2 & 2.2 & 0.182 \\
\hline 10. Dizziness & 1.5 & 2.2 & 1.3 & 1.6 & 0.012 \\
\hline 11. Ear pain & 1.1 & 1.8 & 0.9 & 1.2 & 0.003 \\
\hline 12. Facial pain/pressure & 2.2 & 3.2 & 1.6 & 2.8 & 0.0001 \\
\hline 13. Difficulty falling asleep & 1.8 & 2.2 & 1.6 & 2.2 & 0.134 \\
\hline 14. Waking up at night & 2.3 & 2.4 & 2.3 & 2.5 & 0.753 \\
\hline 15. Lack of a good night's sleep & 2.4 & 2.7 & 2.3 & 2.4 & 0.391 \\
\hline 16. Waking up tired & 2.7 & 3.2 & 2.5 & 3.0 & 0.076 \\
\hline 17. Fatigue & 2.7 & 3.3 & 2.5 & 2.8 & 0.023 \\
\hline 18. Reduced productivity & 2.4 & 3.1 & 2.1 & 2.4 & 0.009 \\
\hline 19. Reduced concentration & 2.1 & 2.7 & 2.0 & 2.2 & 0.067 \\
\hline 20. Frustrated/restless/irritable & 2.0 & 2.4 & 1.8 & 2.4 & 0.055 \\
\hline 21. Sad & 1.4 & 2.0 & 1.2 & 1.7 & 0.016 \\
\hline 22. Embarrassed & 2.3 & 2.2 & 2.3 & 2.1 & 0.768 \\
\hline
\end{tabular}

$\mathrm{n}$ = number of patients; NP = nasal polyps

${ }^{\mathrm{a}}$ missing data $=27 .{ }^{\mathrm{b}}$ The Kruskall-Wallis test was used to compare the rhinosinusitis groups. Statistically significant differences: $\mathrm{p}<0.05$.

the HAD questionnaire $(r=-0.728, p<0.0001)$.

\section{Absenteeism due to sinus problems}

Over half the patients, $57 \%$, reported absenteeism due to sinus problems (Table 5). There was a significant difference in absenteeism between the rhinosinusitis groups $(\mathrm{p}<0.0001)$; the patients with RARS and CRS-NP reporting significantly higher absenteeism than those with CRS+NP. There was no statistically significant difference in absenteeism when comparing the duration of sinus problems. The distribution of age across the absenteeism groups is accounted for in Table 5.

\section{Polyp grade and HRQOL scores}

The surgeons had graded polyps in 199 patients in the cohort, resulting in a mean value of $1.3 \pm 1.1$. There was a statistically significant positive correlation between the extent of polyps and the scores on 10 of the SNOT-22 questions (need to blow nose, sneezing, runny nose, nasal blockage/congestion, dizziness, ear pain, facial pain/pressure, fatigue, reduced concentration and frustration/restlessness/irritability), but not with the total SNOT-22 score or the VAS score. There was also a significant positive correlation between the scores of the BP and VT domains of SF-36 and the extent of polyps (data not shown).
There was no significant difference in total SNOT-22 score or VAS score when comparing endoscopic polyp extent in terms of NP severity or NP side scores. However, when the items of the questionnaires were analysed separately, differences were revealed. The patients with different NP severity grades (i.e. grade A or B) scored statistically significantly differently (p < $0.05)$ in the SNOT-22 questions: need to blow nose, sneezing, runny nose, loss of sense of taste/smell, facial pain/pressure, frustration/restlessness/irritability and the BP and VT subscales. The patients with different NP side grades (i.e. grades I - V) scored significantly differently in the SNOT-22 questions loss of sense of taste/smell, coughing, ear pain, facial pain/pressure and the BP subscale. Scores for the SNOT-22 questions on loss of sense of smell/taste and facial pain or pressure were the most sensitive to differences in, and correlations with, all kinds of polyp grades $(\mathrm{p}<0.0001)$. There was no statistically significant difference in scoring of the SNOT questions on sleep between patients with unilateral and bilateral nasal obstruction caused by polyps. We found no significant difference in HAD scoring when comparing different kinds of polyp grading.

\section{DISCUSSION}

This prospective study shows that patients with RARS, CRS+NP and CRS-NP awaiting surgery have significantly 
Table 3. Outcome measures of SF-36 with comparisons between the three rhinosinusitis groups.

\begin{tabular}{|c|c|c|c|c|c|c|}
\hline & \multicolumn{2}{|c|}{ All patients } & \multirow{2}{*}{$\begin{array}{c}\text { Recurrent acute } \\
\text { rhinosinusitis } \\
\text { Mean }\end{array}$} & \multirow{2}{*}{$\begin{array}{c}\text { Chronic } \\
\text { rhinosinusitis } \\
\text { with NP }\end{array}$} & \multirow{2}{*}{$\begin{array}{c}\text { Chronic } \\
\text { rhinosinusitis } \\
\text { without NP } \\
\text { Mean }\end{array}$} & \multirow[t]{2}{*}{$p$ value $^{a}$} \\
\hline & Mean & $\mathrm{CI}^{\mathrm{w}}$ & & & & \\
\hline Physical functioning & 80.5 & $77.6-83.3$ & 76.8 & 82.5 & 76.6 & 0.081 \\
\hline Role physical & 62.4 & $56.8-68.1$ & 49.2 & 66.4 & 59.9 & 0.062 \\
\hline Bodily pain & 63.2 & $59.1-67.3$ & 48.8 & 70.6 & 49.7 & 0.0001 \\
\hline General health & 60.8 & $57.6-63.9$ & 58.8 & 62.6 & 56.1 & 0.305 \\
\hline Vitality & 51.5 & $48.0-55.0$ & 45.1 & 54.6 & 45.9 & 0.070 \\
\hline Social functioning & 72.8 & $69.3-76.3$ & 69.3 & 74.3 & 70.4 & 0.436 \\
\hline Role emotional & 71.0 & $65.5-76.6$ & 65.6 & 72.6 & 70.2 & 0.586 \\
\hline Mental health & 73.1 & $70.4-75.9$ & 69.2 & 74.7 & 71.1 & 0.096 \\
\hline Physical health domain & 44.5 & $43.0-45.9$ & 40.7 & 46.2 & 41.5 & 0.002 \\
\hline Mental health domain & 43.9 & $42.2-45.6$ & 42.7 & 44.3 & 43.6 & 0.852 \\
\hline
\end{tabular}

NP: nasal polyps

${ }^{a}$ The Kruskall-Wallis test was used to compare patient groups with recurrent acute rhinosinusitis, chronic rhinosinusitis with and without nasal po lyps, respectively. Statistically significant differences: $\mathrm{p}<0.05 .{ }^{\mathrm{b}} 95 \%$ confidence interval.

Table 4. HAD scores for the three rhinosinusitis groups.

\begin{tabular}{|c|c|c|c|c|}
\hline & $\begin{array}{l}\text { All patients } \\
(\mathrm{n}=205)\end{array}$ & $\begin{array}{l}\text { Recurrent acute } \\
\text { rhinosinusitis } \\
(\mathrm{n}=34)\end{array}$ & $\begin{array}{l}\text { Chronic rhinosi- } \\
\text { nusitis with NP } \\
\quad(\mathrm{n}=133)\end{array}$ & $\begin{array}{c}\text { Chronic rhinosinusitis } \\
\text { without NP } \\
(\mathrm{n}=38)\end{array}$ \\
\hline Possible anxiety ${ }^{a}$ & $27(13 \%)$ & $5(15 \%)$ & $16(12 \%)$ & $6(16 \%)$ \\
\hline Probable anxiety ${ }^{\mathrm{b}}$ & $25(12 \%)$ & $5(15 \%)$ & $14(10 \%)$ & $6(16 \%)$ \\
\hline Possible depression ${ }^{\mathrm{c}}$ & $20(10 \%)$ & $3(9 \%)$ & $10(7 \%)$ & $7(18 \%)$ \\
\hline Probable depression $^{\mathrm{d}}$ & $8(4 \%)$ & $2(6 \%)$ & $5(4 \%)$ & $1(3 \%)$ \\
\hline Number of patients scoring $>7$ points $^{\mathrm{e}}$ & $57(28 \%)$ & $11(32 \%)$ & $33(25 \%)$ & $13(34 \%)$ \\
\hline Mean anxiety score & 4.9 & 6.2 & 4.4 & 5.5 \\
\hline Mean depression score & 3.9 & 3.9 & 3.7 & 4.6 \\
\hline
\end{tabular}

$\mathrm{n}$ : number of patients answering the questionnaire; NP: nasal polyps.

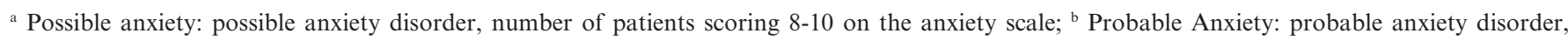
number of patients scoring $>10$ on the anxiety scale; ${ }^{\mathrm{c}}$ Possible Depression: possible depression disorder, number of patients scoring $8-10$ on the depression scale; ${ }^{\mathrm{d}}$ Probable Depression: probable depression disorder, number of patients scoring $>10$ on the depression scale. ${ }^{\mathrm{e}}$ number of patients scoring $>7$ points: number of patients scoring $>7$ on at least one scale; the patient is only counted once even if the scores exceed 7 on both scales.

Table 5. Number of patients reporting absenteeism due to sinus problems in the three rhinosinusitis groups.

\begin{tabular}{lcccccc}
\hline & \multicolumn{4}{c}{ Absenteeism } \\
& 0 days & $1-7$ days & $8-14$ days & $15-30$ days & $>30$ days & Total \\
\hline Recurrent acute rhinosinusitis & 6 & 4 & 6 & 2 & 26 \\
Chronic rhinosinusitis with NP & 60 & 24 & 14 & 6 & 9 & 113 \\
Chronic rhinosinusitis without NP & 9 & 6 & 6 & 9 & 4 & 34 \\
Total & $75(43 \%)$ & $34(20 \%)$ & $26(15 \%)$ & $17(10 \%)$ & $21(12 \%)$ & 173 a \\
Age in years median (min-max) & $54(41-62)$ & $41(34-54)$ & $40(35-54)$ & $51(41-56)$ & $42(32-53)$ & $48(18-85)$ \\
\hline
\end{tabular}

NP: nasal polyps.

${ }^{a}$ Missing data $=34$

The Kruskal-Wallis test was used to compare all groups $(\mathrm{p}<0.0001)$ followed by the Mann-Whitney $\mathrm{U}$ test: Recurrent acute rhinosinusitis vs. chronic rhinosinusitis with nasal polyps ( $<0.0001$ ), Recurrent acute rhinosinusitis vs. chronic rhinosinusitis without nasal polyps $(p=0.46)$, Chronic rhinosinusitis without nasal polyps vs. chronic rhinosinusitis with nasal polyps $(\mathrm{p}=0.001)$. 
lower SF-36 scores than the normal Swedish normal population, which results in a considerable degree of absenteeism from work. There was statistical evidence of differences between these three subgroups regarding HRQOL scoring when analysing the items and subscales of the questionnaires separately. The Swedish version of SNOT-22 was found to be easy to use and to have a strong internal consistency. Significant differences in patient-reported absenteeism caused by sinus problems were found between patients with RARS, CRS+NP and CRS-NP. To the best of our knowledge, this is the first report of its kind on CRS with or without NP using the $\mathrm{EP}^{3} \mathrm{OS}$ criteria.

Since this study started, interest in HRQOL as an outcome measure has increased, and other studies on the impact of rhinosinusitis on HRQOL have been performed. The largest study published by far is Hopkins et al.'s National Comparative Audit in the UK ${ }^{(7)}$, in which patient and operative characteristics as well as patient outcomes of 3,128 patients are described up to 36 months after surgery. The patients in our study had statistically significantly higher preoperative SNOT-22 scores (mean 51.8 vs. 42.0 in the UK Audit), indicating a lower HRQOL than in the English cohort. This could, as a suggestion, be because Swedish patients are operated on later in the course of their disease, and/or that defined criteria for RARS and CRS $\pm N P$ were used in the present study. The mean SNOT-22 score of healthy patients in the UK Audit was $9.3^{(25)}$, confirming that chronic sinus problems significantly affect HRQOL when measured with the SNOT-22 questionnaire. We found high internal consistency in the Swedish SNOT-22 questionnaire (Cronbach's alpha of 0.91), which is of the exactly same level as in the UK audit ${ }^{(14)}$, and on the same level as previously reported for the same questionnaire in other languages (Cronbach's alpha of $\left.0.83-0.86^{(15,17,18)}\right)$.

As normative values for SF-36 differ between different populations, direct comparisons of HRQOL scoring between cohorts is unreliable. However, there is a great conformity of the health profile and SF-36 scores of the normal populations the US, the UK and Sweden according to studies (20), demonstrating a good discriminative capacity in gender and age. Gliklich and Metson ${ }^{(1)}$ described the impact on health of CRS in patients seeking otorhinolaryngological care and found significantly lower SF-36 scores in five of eight domains compared with the normal US population. In that study there was no subgrouping of the CRS patients, and more than $80 \%$ subsequently underwent surgery ${ }^{(1)}$. Other studies performed on patients undergoing surgery have also found CRS to affect HRQOL measures using the SF-36 questionnaire ${ }^{(26-28)}$, showing improvement in scoring after surgery. The patients in our study scored statistically significantly lower in all eight SF-36 domains than the normal Swedish population. It should be noted that EP3OS criteria were not used to define the patient groups in any of the above mentioned studies.
Durr et al., separated patients with RARS from other patients with chronic rhinosinusitis ${ }^{(29)}$ using the same definition of RARS as that used in the present study. They found statistically significant differences $(p<0.05)$ between the three rhinosinusitis groups in the BP subscale only. In a Swedish study performed by Olsson and co-workers ${ }^{(30)}$ it was found that HRQOL was significantly impaired in patients with NP and asthma. In our study, the presence of NP implied statistically significantly higher scores of BP in SF-36 ( $p<$ $0.000)$, reflecting less pain. The fact that pain seems to be less of a problem in patients with NP is not unexpected, as personal clinical experience has shown that patients with RARS and CRS-NP report pain more often. The cohort in the present study had generally lower scores in SF-36 than Swedish patients with asthma ${ }^{(31)}$, ulcerative colitis and Crohn's disease $^{(32)}$. Thus, chronic sinus disease appears to have a greater impact on HRQOL than other severe chronic conditions in Sweden. Even long-term cancer survivors seem to report less impact on HRQOL, measured by SF-36 ${ }^{(33)}$, than patients with RARS and CRS \pm NP referred for surgery.

In the present study, the rate of depression was $14 \%$ and the rate of anxiety $25 \%$, according to the patients' scores in the HAD questionnaire. The mean HAD scores were similar to those of a cohort consisting of normal members of the Swedish population ${ }^{(34)}$, but in that study the drop-out rate was greater than $50 \%$. In a study presented by Davis and co-workers ${ }^{(35)}$, using another questionnaire to screen for psychiatric morbidity, $25 \%$ of patients with CRS presenting for sinus surgery screened positive for depressive disorders and $17 \%$ for anxiety. The frequency of scoring $>7$ points on the HAD scale was lower in our cohort than in Swedish patients with a diagnosis of head and neck cancer ${ }^{(22)}$ (28\% compared with $36 \%$ in cancer patients at diagnosis), which could be expected. Psychiatric morbidity does not seem to be overrepresented in patients with RARS or CRS \pm NP awaiting surgery. As HAD scoring is strongly correlated to mental health scoring in SF-36, we recommend the use of HAD before referring patients for surgery, to be able treat underlying depression or anxiety.

The cohort in the present study was divided into the three groups: mild, moderate and severe symptoms, according to an established VAS method ${ }^{(23)}$. More than $90 \%$ of the patients in the present study suffered from severe or moderate disease, according to the VAS scores, indicating advanced sinus disease. The higher the VAS score, the worse the patient scored in SNOT-22, all eight subscales of SF-36 and HAD. All these correlations were strongly statistically significant, as described above. Consequently, the single question, 'How troublesome are your symptoms of rhinosinusitis?,' can be used as a simple tool to assess the severity of sinus disease and thus the degree of HRQOL. As stated in the EP ${ }^{3} \mathrm{OS}$ document, surgery should be the treatment of choice only in severe cases. Thus, the use of a single question could provide an easy method of assessing indications for surgery. Additionally, the evaluation of differ- 
ent forms of treatment is of great interest today, and this simple instrument could provide a means of assessing the quality of treatment in clinical rhinological practice.

There have been reports on the cost to society of chronic rhinosinusitis. Two of these reports are based on patient-reported diagnosis ${ }^{(4)}$ and estimates of sick-leave based on SF-36 scores ${ }^{(36)}$, respectively. Hellgren et al. published a report on absenteeism caused by rhinitis in $2009^{(37)}$. They reported a mean productivity loss of 5.1 days or $€ 653$ per worker per year, as the result of allergic rhinitis and common colds. The findings of their study are not directly comparable to the results of our study, since the patients in our cohort were asked to choose one of five intervals of absenteeism. However, the patients with RARS, CRS+NP and CRS-NP in this study reported a higher degree of absenteeism. Calculating the mean of these scores, gives a rate of absenteeism caused by sinus problems somewhere between 8 and 14 days per year. The presence of nasal polyps implied less sick-leave in the present study.

To investigate the impact of the extent of endoscopically graded polyps on patient-perceived HRQOL, we analysed the patients into three different groups according to the extent of polyps, as described above. No statistically significant correlation was found between the polyp grades and the total SNOT-22 score or the VAS score, but significant correlations were seen between polyp grade and several of the separate HRQOL items. We therefore recommend analysis of separate items or subscales in HRQOL measures, to identify differences in HRQOL scoring, when studying different kinds of subgroups in sinus disease. The SNOT-22 questions on loss of sense of smell/taste and facial pain or pressure were found to be the items most correlated to endoscopic polyp extent. The higher the polyp grade, the more loss of sense of smell/taste, but the less facial pain /pressure. It is interesting that no differences in sleeping measured by SNOT22 (questions 13 - 15) were seen when comparing unilateral and bilateral nasal obstruction caused by polyps. Furthermore, scoring of the SNOT-22 question on feeling sense of nasal obstruction was not correlated to endoscopic polyp grading.

One shortcoming of this study could be that all patients eligible for inclusion during the study period were not reported. We cannot rule out that some non-random selection bias has occurred. The patients who did not respond to the questionnaires might have had worse or better outcome than those who did chose to participate in the survey. It is also possible that the data missing in the questionnaires were different from those that were collected and analysed. This was an observational study reflecting the status of patients with RARS and $\mathrm{CRS} \pm \mathrm{NP}$ as treated in Sweden according to current guidelines and practices. It should be noted that, the patients in our study had already been given suitable medication, and had been referred for FESS when included in the study. Thus, these patients could be considered to have more sinus problems than the average patient with chronic sinus disease in Sweden. This is supported by the finding that $53 \%$ of the patients in this study were found to suffer form severe disease according to the VAS classification. As stated in EP3OS, these are the patients who should be offered surgery. Consequently, the patients in this study were highly likely to have had worse HRQOL scores and reported a higher degree of absenteeism than the average Swedish patient with RARS, CRS+NP or CRS-NP. Despite the concerns described above, it should be emphasized that we have presented data from prospectively included patients, from both university and regional hospitals, diagnosed using uniform criteria from the $\mathrm{EP}^{3} \mathrm{OS}$ document, and that the size of the cohort is considerable.

\section{CONCLUSIONS}

This prospective study shows that RARS and CRS $\pm N P$ referred for FESS have significant impact on HRQOL; in some cases, greater than that of other severe chronic conditions. A single question rated on a VAS seems to offer a simple but effective estimate of the severity of chronic sinus disease, and could be used to decide whether surgery is indicated or not. The Swedish version of SNOT-22 was shown to have strong internal consistency, but it may be appropriate to consider the items of this questionnaire separately when comparing different groups of patients. Our findings regarding absenteeism due to rhinosinusitis could be of importance as they give an indication of the cost to society associated with this condition. We suggest that further studies be carried out on absenteeism resulting from rhinosinusitis. Studies are in progress to evaluate how much HRQOL scoring improve after FESS, if preoperative HRQOL scoring have an impact on the surgical outcome and if FESS can decrease absenteeism from work.

\section{ACKNOWLEDGEMENTS}

This study was performed with grants from the Swedish ACTA Foundation and Skåne county council's research and development foundation. The authors also wish to thank:

Wytske Fokkens, MD, PhD, Department of Oto-RhinoLaryngology, Amsterdam Medical Centre, Amsterdam, the Netherlands, for her guidance in designing this study; Peter Höglund, MD, PhD, Department of Clinical Chemistry and Pharmacology, Lund University, Lund, Sweden, for help with the statistical analysis; as well as the otorhinalaryngologists participating in this study, especially Pernilla Björnberg, MD, Department of Oto-Rhino-Laryngology, Head and Neck Surgery, Skåne University Hospital, Malmö, Sweden; Maria Hillerström, MD, PhD, Department of Oto-RhinoLaryngology, Uppsala University Hospital, Uppsala, Sweden; Magnus Ivarsson, MD, PhD, Department of Oto-RhinoLaryngology, Sahlgrenska University Hospital, Gothenburg, Sweden, and Mattias Jangaard, MD, Department of OtoRhino-Laryngology, Karolinska University Hospital, Solna, Sweden.

\section{AUTHORSHIP CONTRIBUTION}

PSJ contributed to the conception and design of the study, the acquisition, analysis and interpretation of data and manuscript 
writing. BO contributed to the analysis and interpretation of data and the critical revision of the manuscript. $\mathrm{CvB}$ and $\mathrm{MJ}$ contributed to the conception and design of the study and to the critical revision of the manuscript. MAE contributed to the conception and design of the study, the analysis and interpretation of the data and critical revision of the manuscript. All five authors have read and approved the final version of the manuscript.

\section{CONFLICT OF INTEREST}

None

\section{REFERENCES}

1. Gliklich RE, Metson R. The health impact of chronic sinusitis in patients seeking otolaryngologic care. Otolaryngol Head Neck Surg. 1995; 113: 104-109.

2. Tomassen P, Newson RB, Hoffmans R, et al. Reliability of EP3OS symptom criteria and nasal endoscopy in the assessment of chronic rhinosinusitis - a GA(2)LEN study. Allergy. 2011; 66: 556-561.

3. Osguthorpe JD. Surgical outcomes in rhinosinusitis: what we know. Otolaryngol Head Neck Surg. 1999; 120: 451-453.

4. Adams PF, Hendershot GE, Marano MA. Current estimates from the National Health Interview Survey, 1996. Vital Health Stat. 10. 1999; 1-203.

5. Fokkens W, Lund V, Bachert C, et al. EAACI position paper on rhinosinusitis and nasal polyps executive summary. Allergy. 2005; 60: 583-601.

6. Fokkens W, Lund V, Mullol J. European position paper on rhinosinusitis and nasal polyps 2007. Rhinology Suppl. 20; 1-136.

7. Hopkins C, Browne JP, Slack R, et al. The national comparative audit of surgery for nasal polyposis and chronic rhinosinusitis. Clin Otolaryngol. 2006; 31: 390-398.

8. Gliklich RE, Metson R. Effect of sinus surgery on quality of life. Otolaryngol Head Neck Surg. 1997; 117: 12-17.

9. Sahlstrand-Johnson P, Buchwald C. Outcome in Functional Endoscopic Sinus Surgery (FESS) for Rhinosinusitis. Journal of ENT Masterclass Year Book 2009. Review. 2009; 2: 78-82.

10. Lanza DC, Kennedy DW. Adult rhinosinusitis defined. Otolaryngol Head Neck Surg. 1997; 117(3 Pt 2): S1-7.

11. Bhattacharyya N, Lee KH. Chronic recurrent rhinosinusitis: disease severity and clinical characterization. Laryngoscope. 2005; 115: 306310.

12. Piccirillo JF, Merritt MG, Jr., Richards ML. Psychometric and clinimetric validity of the 20-Item Sino-Nasal Outcome Test (SNOT-20). Otolaryngol Head Neck Surg. 2002; 126: 41-47.

13. Morley AD, Sharp HR. A review of sinonasal outcome scoring systems - which is best? Clin Otolaryngol. 2006; 31: 103-109.

14. Hopkins C, Gillett S, Slack R, Lund VJ, Browne JP. Psychometric validity of the 22-item Sinonasal Outcome Test. Clin Otolaryngol. 2009; 34: 447-454.

15. Schalek P, Otruba L, Hahn A. Quality of life in patients with chronic rhinosinusitis: a validation of the Czech version of SNOT-22 questionnaire. Eur Arch Otorhinolaryngol. 2010; 267: 473-475.

16. Lu W, Qi F, Gao ZQ, Feng GD, Yuan XD, Jin XF. Quality of life survey on patients with chronic rhinosinusitis by using Chinese version of the 22-item sinonasal outcome test (SNOT-22). Zhonghua Er Bi Yan Hou Tou Jing Wai Ke Za Zhi. 2008; 43: 18-21.

17. Baumann I, Blumenstock G, DeMaddalena H, Piccirillo JF, Plinkert PK. Quality of life in patients with chronic rhinosinusitis: validation of the Sino-Nasal Outcome Test-20 German Adapted Version]. HNO. 2007; 55: 42-47.

18. Lange B, Thilsing T, Al-Kalemji A, Baelum J, Martinussen T, Kjeldsen A. The Sino-Nasal Outcome Test 22 validated for Danish patients. Dan Med Bull. 2011; 58: A4235.

19. Fayers P, Machin D. Quality of life: the assessment, analysis, and interpretation of patient-reported outcomes. 2nd ed. Chichester: John Wiley \& Sons Ltd; 2007.

20. Sullivan M, Karlsson J, Taft C. SF-36: Swedish manual and inter- pretation guide, 2nd Edition. Gothenburg: Sahlgrenska University Hospital. 2002.

21. Zigmond AS, Snaith RP. The hospital anxiety and depression scale. Acta Scand. 1983; 67: 361-370.

22. Hammerlid E, Ahlner-Elmqvist M, Bjordal K, et al. A prospective multicentre study in Sweden and Norway of mental distress and psychiatric morbidity in head and neck cancer patients. Br J Cancer. 1999; 80: 766-774

23. Lim M, Lew-Gor S, Darby Y, Brookes N, Scadding G, Lund VJ. The relationship between subjective assessment instruments in chronic rhinosinusitis. Rhinology. 2007; 45: 144-147.

24. Sullivan M, Karlsson J. The Swedish SF-36 Health Survey III. Evaluation of -based validity: results from normative population. J Clin Epidemiol. 1998; 51: 1105-1113.

25. Gillett S, Hopkins C, Slack R, Browne JP. A pilot study of the SNOT 22 score in adults with no sinonasal disease. Clin Otolaryngol. 2009; 34: 467-469.

26. Ragab SM, Lund VJ, Scadding G, Saleh HA, Khalifa MA. Impact of chronic therapy on quality of life: a prospective randomized controlled trial. Rhinology. 2010; 48: 305-311.

27. Smith TL, Litvack JR, Hwang PH, et al. Determinants of outcomes of sinus: a multi-institutional prospective cohort study. Otolaryngol Head Neck Surg. 2010; 142: 55-63.

28. Winstead W, Barnett SN. Impact of endoscopic sinus surgery on global health: an outcomes study. Otolaryngol Head Neck Surg. 1998; 119: 486-491.

29. Durr DG, Desrosiers MY, Dassa C. Impact of rhinosinusitis in health care delivery: the Quebec experience. J Otolaryngol. 2001; 30: 93-97.

30. Olsson P, Ehnhage A, Nordin S, Stjarne P. Quality of life is improved by surgery and fluticasone in nasal polyposis with asthma. Rhinology. 2010; 48: 325-330.

31. Larsson U, Taft C, Karlsson J, Sullivan M. Gender and age differences in the relative burden of rhinitis and asthma on health-related quality of life--a Swedish population study. Respir Med. 2007; 101: 1291-1298.

32. Nordin K, Pahlman L, Larsson K, Sundberg-Hjelm M, Loof L. Health-related quality of life and psychological distress in a population-based sample of Swedish patients with inflammatory bowel disease. Scand J Gastroenterol. 2002; 37: 450-457.

33. Hammerlid E, Taft C. Health-related quality of life in long-term head and neck cancer survivors: a comparison with general population norms. Br J Cancer. 2001; 84: 149-156.

34. Lisspers J, Nygren A, Soderman E. Hospital Anxiety and Depression Scale (HAD): some psychometric data for a Swedish sample. Acta Psychiatr Scand. 1997; 96: 281-286.

35. Davis GE, Yueh B, Walker E, Katon W, Koepsell TD, Weymuller EA. Psychiatric distress amplifies symptoms after surgery for chronic rhinosinusitis. Otolaryngol Head Neck Surg. 2005; 132: 189-196.

36. Gliklich RE, Metson R. Economic implications of chronic sinusitis. Otolaryngol Head Neck Surg. 1998; 118 (3 Pt 1): 344-349.

37. Hellgren J, Cervin A, Nordling S, Bergman A, Cardell LO. Allergic rhinitis and the common cold-high cost to society. Allergy. 2010; 65: 776-783.

Pernilla Sahlstrand Johnson, MD

Consultant Otorhinolaryngologist

Faculty of Medicine, Lund University

Department of Oto-Rhino-Laryngology

Skåne University Hospital, Malmö

SE-205 02 Malmo

Sweden

Tel.: +46-40-332 334

Fax: +46-40-336 229

E-mail: pernilla.sahlstrand_johnson@med.lu.se 